\title{
Cataract surgery in patients with pseudoex- foliation syndrome: current updates
}

\author{
This article was published in the following Dove Press journal: \\ Clinical Ophthalmology \\ 31 July 2017 \\ Number of times this article has been viewed
}

\section{Luigi Fontana \\ Marco Coassin \\ Alfonso lovieno \\ Antonio Moramarco \\ Luca Cimino}

Ophthalmology Unit, Arcispedale Santa Maria Nuova - IRCCS, Reggio

Emilia, Italy
Correspondence: Luigi Fontana Ophthalmic Unit, Arcispedale Santa Maria Nuova - IRCCS, Viale Risorgimento 80, 42010 Reggio Emilia, Italy Email luifonta@gmail.com

\begin{abstract}
Pseudoexfoliation is a ubiquitous syndrome of multifactorial origin affecting elderly people by increasing the risk of cataract and secondary glaucoma development. Despite modern techniques and technologies for cataract surgery, pseudoexfoliation syndrome represents a challenge for surgeons because of the increased weakness of the zonular apparatus and limited pupil dilation. Due to the inherent difficulties during surgery, the risk of vitreous loss in these patients is several times higher than in cataract patients without pseudoexfoliation. Using currently available surgical devices (ophthalmic viscosurgical device, iris retractors and ring dilators, capsular tension ring, etc.), the risk of intraoperative complications may be much reduced, allowing the surgeon to handle difficult cases with greater confidence and safety. This review analyzes the methodologic approach to the patient with zonular laxity with the aim of providing useful advices to limit the risks of intraoperative and postoperative complications. From the preoperative planning, to the intraoperative management of the small pupil and phacodonesis, and to the postoperative correction of capsule phimosis and intraocular lens dislocation, a step approach to the surgical management of pseudoexfoliation patients is illustrated.
\end{abstract}

Keywords: pseudoexfoliation syndrome, cataract surgery, zonular laxity, intraocular lens implant, complications

\section{Introduction}

Cataract still remains the world's leading cause of blindness and visual impairment in the elderly population, despite the decreasing number of people affected. ${ }^{1}$ If age is the main risk factor for cataract progression, pseudoexfoliation syndrome (PXF) represents an independent additional hazard for the development of nuclear sclerosis and indication for cataract surgery. ${ }^{2,3}$

PXF syndrome is a multifactorial, genetically determined, age-related and environmentally influenced disorder of the elastic fiber structure, characterized by excessive production and accumulation of an elastotic material within a multitude of intra and extraocular tissues. ${ }^{4}$ For this reason, PXF is a diffuse disease with ocular and systemic manifestations.

In all populations, the prevalence of PXF increases markedly with age as the deposition of the typical white fibrillar material, that characterizes this disease, progressively accumulates during life. With an occurrence as low as $0 \%$ in the middle age group (49-54 years), this value increases up to $6.25 \%$ among elderly patients of 85 years or more in the Australian population ${ }^{2}$ and up to $5 \%$ in the USA, ${ }^{5}$ with both men and women equally affected. Also known as the "Viking disease", PXF is more common in Scandinavia and in other Northern Europeans with low climatic temperature, though the disease is reported in all population types and races. ${ }^{6,7}$ It is estimated 
that in Sweden, an 87-year-old individual has $61 \%$ chance of having PXF. ${ }^{8}$

Pseudoexfoliation is diagnosed by the deposition of white, "dandruff-like", fluffy material, virtually in all the structures of the human eye, but more importantly in the anterior segment: corneal endothelium, anterior capsule, lens zonules, iris, and trabecular meshwork. The material is composed of amyloid, laminin, elastic fibers, collagen, and basement membrane., ${ }^{9,10}$ The same material seen in the ocular district has been found in other parts of the human body such as heart, lung, liver, kidney, cerebral meninges, and blood vessels, ${ }^{11}$ indicating that PXF is a multiorgan disease. This may explain why patients with ocular PXF may present a history of systemic hypertension, abdominal aorta aneurysm, angina, cardiovascular disease, and stroke. ${ }^{12,13}$ Despite all these, life expectancy does not differ between persons with and without PXF. ${ }^{14}$

The pathogenesis of PXF is multifactorial, where geographical and environmental factors, together with genetic predisposition, explain the different incidence of this syndrome across the world. A documented association with mutations in the lysyl oxidase-like 1 gene (LOXL1) at the locus $15 q 22,{ }^{15}$ which codes for elastic fiber components of extracellular matrix, suggests a genetic factor for the inheritance of this disease. For this reason, it has been suggested that PXF is a form of elastosis resulting from the overproduction of elastic microfibrillar components such as fibrillin-1. ${ }^{16}$ The systemic origin of the disease explains why patients with unilateral involvement manifest PXF signs in the normal fellow eye at a later time, indicating that PXF is a generalized, bilateral disorder with a markedly asymmetric clinical presentation at onset. Patients with unilateral signs of the disease are usually younger than those with bilateral involvement.

PXF is generally recognized late in life as the course of the disease is for long time subclinical and the degree of ocular involvement and visual loss is often asymmetrical or more rarely unilateral. The diagnosis of PXF is of paramount importance as this disease is a major risk factor for complications during cataract surgery and the most frequent cause of secondary glaucoma. ${ }^{17}$ Intraoperative and postoperative complications may arise from the weakened capsule and zonular apparatus (zonulopathy) secondary to the progressive proteolytic disintegration of the suspensory ligament, responsible for the instability of the crystalline lens during surgery, capsular rupture, zonular dialysis, vitreous loss, nuclear luxation, decentration or dislocation of the intraocular lens (IOL) with time. Further difficulties during surgery result from poor or inadequate pupil dilation (iridopathy), secondary to atrophic changes of the iris sphincter and stroma characterized by transillumination defects. Postoperative anterior chamber inflammation and fibrinous reaction occur frequently in PXF patients due to an acquired weakened blood-aqueous barrier. ${ }^{18}$ Corneal endothelial morphological and functional changes (endotheliopathy) are seen in eyes affected with PXF, explaining the greater susceptibility of these eyes to surgical trauma, resulting in transitory and permanent corneal decompensation. ${ }^{19}$

Modern techniques and instruments for cataract surgery have profoundly improved the confidence toward the surgical approach and operative management of PXF cases; however, the higher risk of complications with surgery still suggests the need for greater expertise and surgical skill for these patients. ${ }^{17}$ The aim of this paper is to review the surgical approach to the pseudoexfoliative cataract, in light of current scientific knowledge and modern surgical techniques, specifically addressing the management of patients at preoperative, intraoperative, and postoperative steps.

\section{Preoperative}

The signs of PXF can be identified on slit-lamp examination. Biomicroscopy, after pupil dilation, has a high sensitivity and specificity on recognizing the presence of fibrillar material over the lens surface and iris even at early stages of the disease. The diagnosis of PXF is based on slit lamp observation of the typical gray-white flakes at the pupillary margin and fibrillary deposits over the anterior lens capsule, mostly concentrated in the pupillary area, often separated from the peripheral residues by a clear zone (Figure 1). Sometimes

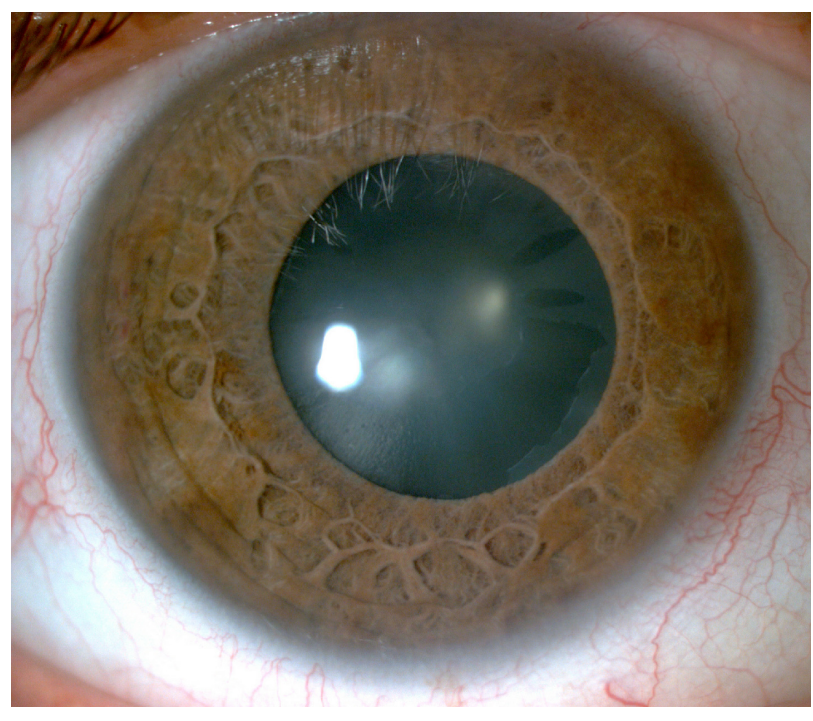

Figure I Slit lamp photograph showing signs of cataract and pseudoexfoliation. Notes: The lens surface shows sign of exfoliating fibrillar material with accumulation of whitish flakes at the pupillary margin. The pupil is only moderately dilated after pharmacological mydriasis. 
in the presence of an advanced cataract, with a dimmed red reflex, signs of PXF are more difficult to identify as the whitish flakes do not contrast with the color of the lens. Once the diagnosis of cataract and the indication for surgery have been established, signs of reduced zonular integrity should be explored in order to assess the risk of complications during surgery. The degree of zonular laxity does not strictly correlate with the amount and extension of pseudoexfoliative material visible over the lens surface, but more with the age of the patient. Typical signs of zonular weakness are phacodonesis, with eye movements, and sometimes a reduction of the anterior chamber depth due to a forward shift of the lens. Changes in anterior chamber depth may be localized when zonular dehiscence develops involving only some hours of the capsular lens ligament. The incidence of phacodonesis and/or lens subluxation has been reported between $8.4 \%$ and $10 \%$ in PXF patients. ${ }^{20}$

Iridodonesis in the presence of phacodonesis is often less remarkable in PXF probably due to an increased rigidity of the ischemic atrophic iris. ${ }^{21}$ Poor pupil dilation is expected in most patients with PXF due to extracellular infiltration and degeneration of the dilator and sphincter muscles with atrophy of the pigment epithelium and stroma. ${ }^{22}$ In some cases, the formation of posterior synechiae further reduces the response to mydriatics. ${ }^{23}$ The degree of maximum pupil dilation should be addressed preoperatively to ensure adoption of appropriate surgical measures. Among these, preoperative nonsteroidal anti-inflammatory drugs (NSAIDs) may be given to help maintain maximum achievable pupil dilation during surgery. ${ }^{24}$

Specular microscopy studies in eyes with and without PXF reported an endothelial cell count 5\% to 9\% lower than in age-matched controls. ${ }^{19,25}$ In PXF patients, not only the cell density but also the cell morphology is altered with a decrease of cell hexagonality and an increase of coefficient of variation. PXF endotheliopathy may result from several causes of direct and indirect damage to the endothelial cells. Accumulation of PXF material between endothelial cells and Descemet's membrane is a possible cause of direct cellular degeneration, ${ }^{26,27}$ whereas elevated intraocular, iris ischemia and increased flare intensity, caused by blood-aqueous barrier breakdown, may indirectly alter the homeostasis of endothelial cells. ${ }^{28,29}$ For the above-mentioned reasons, variations of endothelial cell morphology and density are expected to be funded preoperatively in patients with PXF, and they may be responsible for an increased risk of corneal decompensation after surgery. ${ }^{30}$

Raised intraocular pressure is frequently expected in patients with PXF and the prevalence of glaucoma has been reported as high as $26 \%$ and up to $49 \%$ at the time of cataract surgery. ${ }^{31,32} \mathrm{PXF}$ is the most frequent cause of secondary glaucoma as the results of an increased outflow resistance due to the chronic deposition of pigmented material freed from iris and in addition of exfoliative material throughout the trabecular meshwork, Schlemm canal and collector channels. ${ }^{33}$ The estimated incidence of glaucoma in PXF patients is three to four times greater in patients with than without PXF. ${ }^{34,35}$ Owing to the high level of the intraocular pressure exhibited in these patients, the risk of glaucoma development and progression is elevated. ${ }^{36} \mathrm{Kim}$ et $\mathrm{al}^{37}$ found that PXF patients present a thinner and possibly weaker lamina cribrosa, suggesting a particular susceptibility of these patients to the development of optic nerve cupping. Whether or not cataract surgery may protect against the development of glaucoma in PXF is debatable. According to some studies, the prolonged pressure-lowering effect occurring after surgery may reduce the risk of glaucoma developing over time..$^{38,39}$

\section{Intraoperative}

In the era of phacoemulsification, PXF still represents a relevant challenge for the surgeon. The concurrent presence of poor pupil dilation and zonular weakness is responsible for the increased risk of intraoperative complications that may occur during surgery more frequently than in cataractous eyes without $\operatorname{PXF}^{17}$ (Table 1).

Minimum sufficient pupil dilation $(4-5 \mathrm{~mm})$ may be achieved using a highly viscous cohesive ophthalmic viscosurgical device (OVD) or a combination of two OVDs with different rheological properties. In this case, a dispersive OVD is placed first to coat the corneal endothelium, and then a cohesive OVD is injected centrally to deepen the anterior chamber, flatten the anterior lens capsule, and maintain adequate pupil mydriasis in order to facilitate capsulorhexis creation..$^{40}$ It is worth noting that in the presence of a weak

Table I Preoperative risk factors and intraoperative and postoperative complications in PXF patients with cataract surgery

\begin{tabular}{lll}
\hline Preoperative & Intraoperative & Postoperative \\
\hline Small pupil & Iris damage & Fibrinous uveitis \\
Pupillary synechiae & Posterior capsule tear & Posterior synechiae \\
Zonular laxity/dialysis & Capsular bag dislocation & Capsular phimosis \\
Capsule fragility & Vitreous loss & IOP spike \\
Vitreous prolapse & & IOL in the bag \\
& & dislocation \\
Shallow anterior & & Endothelial cell \\
chamber & damage \\
Hyper deep anterior & & Cystoid macular \\
chamber & & edema \\
Glaucoma & &
\end{tabular}

Abbreviations: IOL, intraocular lens; IOP, intraocular pressure; PXF, pseudoexfoliation syndrome. 
zonular apparatus, over-inflating of the anterior chamber may cause stretching and further damage to the zonules.

Posterior synechiae, if present, should be lysed with a blunt spatula and at the same time pupil may be mechanically stretched using two iris manipulators. Careful manipulation is required due to the ischemic fragile iris in PXF patients. Whenever pupil dilation with OVD provides an insufficient view of the cataract, adequate mydriasis can be achieved using iris hooks or pupillary rings to ensure ample and stable dilation throughout surgery. ${ }^{41}$

The degree of zonular weakness may be assessed as soon as the surgeon begins the capsulorhexis. Difficulties on puncturing the anterior capsule are the first sign of a diminished anterior capsule tension. In this case, a 25-gauge sharp needle bent at the tip may help perform the initial capsulotomy, with minimal pressure applied to the zonular apparatus. Furthermore, in the case of severe zonular weakness, capsule tearing may result due to the lack of resistance forces counteracting the pull of the rhexis forceps, leading to the creation of an excessively small capsulotomy that may complicate phacoemulsification. Using a bimanual technique, counteraction may be achieved with an iris hook, placed to engage the already torn anterior capsule, while forceps continue the capsulorhexis. Ideally, a well-centered 5-6 mm circular continuous capsulorhexis should be adequate to provide access to phacoemulsification maneuvers, IOL support, and implantation of devices that aid in the maintenance of capsular stability during and after surgery.

The presence of whitish scaly material over the lens surface may be mistaken for the anterior capsule layer with the difference that these are typically fragile and tear abnormally when compared with the true anterior capsule. The use of trypan blue to stain the anterior capsule may help recognize this layer when in doubt. ${ }^{42,43}$ Following a complete capsulorhexis, viscodissection, prior to hydrodissection, allows better corticocapsular cleavage than hydrodissection alone, making cortex removal easier and safer by reducing traction on the lens zonules.

Depending on the severity of the zonular instability, the surgeon may use a capsular tension ring (CTR) and/or capsular retractors to support the capsular bag during all steps of phacoemulsification, cortical cleaning, and IOL implantation. In the case of extensive zonular dialysis, more than one device may be employed. Capsule retractors help support the zonular-lens complex in the anteroposterior direction, facilitating hydrodissection and cataract fragmentation, while CTR allows even distension of the capsular bag, supporting areas of zonular dehiscence by redistributing the zonular tension around the capsule and allowing optimal IOL centration. Several reports have shown that CTR implant reduces intraoperative complication in PXF eyes, suggesting the use of this device in cases of mild zonulopathy and phacodonesis. ${ }^{44-46}$ In the case of more extensive zonular weakness, CTR alone may not prevent intraoperative or postoperative late dislocation of the IOL-bag complex. In these cases, capsule retractors should be used during all steps of hydrodissection and phacoemulsification (Figure 2) followed by the implantation of a modified CTR sutured to the sclera to assure anteroposterior stability of the IOL-bag complex. ${ }^{47}$

Phacoemulsification technique is not dissimilar from the one routinely used for other complicated cataract cases. The "chop technique", using vertical and horizontal chopping, allows minimum zonular stress with the advantage of restraining surgical maneuvers to the center of the anterior chamber, avoiding the capsule equator and the pupil. Vitreous prolapse from a zonule defect may be present before surgery or occur during surgery at any time. Limited anterior vitrectomy and tamponade using a cohesive OVD help complete cataract removal and cortex aspiration. Filling the anterior chamber with OVD before removing instruments from the eye prevents collapsing with further anterior herniation of vitreous. In a retrospective comparative study conducted on a large series of cataract procedures, Shingleton et $\mathrm{al}^{48}$ reported a $4 \%$ frequency of vitreous loss in PXF patients compared to $0 \%$ in patients without PXF. Authors of this paper attributed the causes of vitreous loss to zonular weakness rather than capsule tears in these patients. A review study of surgical complications during phacoemulsification reported a $10 \%$ risk of vitreous loss and capsule rupture in PXF patients. ${ }^{17}$

Lens cortex removal may be difficult and traumatic for the capsule and zonules. The presence of a CTR has the

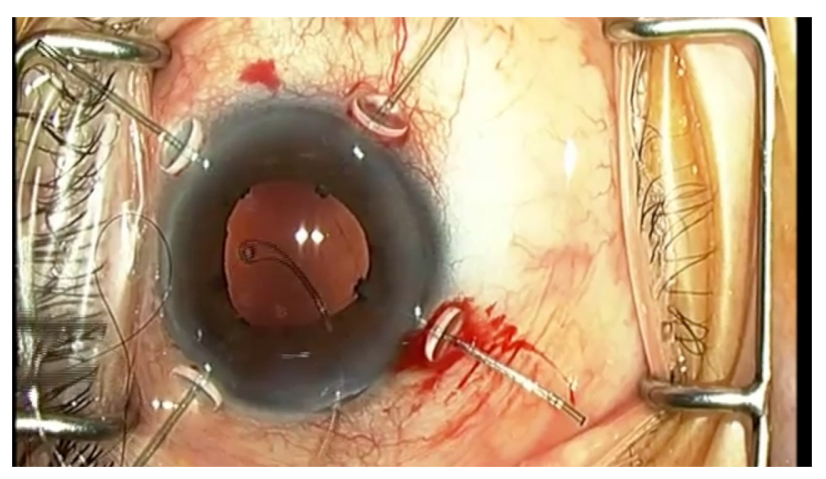

Figure 2 Intraoperative photograph showing capsule retractors stenting the capsular bag during phacoemulsification.

Note: Following cortical lens aspiration, a capsular tension ring is implanted prior to intraocular lens implantation. 
controversial effect of helping cortex removal by stenting the capsular equator, facilitating its separation from the capsule, and at the same time lens cortex may make it more difficult to aspirate when trapped behind a capsular tension device. Profuse and repeated cortical hydration may help soften cortex material and facilitate its aspiration. In all cases, slow-motion careful tangential stripping, in combination with gentle centripetal traction, should allow for accurate cortical removal. ${ }^{49}$

The choice of the IOL to implant in PXF patients is related to the evaluation of the state and future risk of capsular instability. In the presence of zonular weakness, following a CTR implant, a one-piece or three-piece acrylic IOL may be implanted according to the surgeon preference. One-piece IOL requires less manipulation and carries a lower probability of damage to the capsule and zonules and therefore may be considered the IOL of choice in these patients. In consideration of the risk of capsule contraction and IOL decentration, toric IOLs, as well as multifocal IOLs, constitute a possible cause of early or late unsatisfactory visual outcome. Likewise, any IOL placed in the sulcus should be avoided in PXF patients due to the probability of decentration and posterior dislocation. When a complete capsular diaphragm is missing, any anterior chamber angle-supported IOL should not be implanted in consideration of the elevated risk of glaucoma and corneal decompensation secondary to endothelial cell abnormalities.

\section{Postoperative}

A complicated postoperative course may be expected in PXF patients due to the abnormalities of the ocular structures and to the difficulties encountered during surgery (Table 1).

A higher degree of postoperative anterior chamber inflammation is expected in PXF due to the chronic alteration of the blood-aqueous barrier that accompanies the iris ischemic changes seen in these patients. At preoperative, patients with PXF show a baseline level of aqueous protein concentration several times greater than what is measured in cataractous eyes without PXF. ${ }^{50,51}$ Due to the probability of protracted and complicated surgery, with iris manipulation and possible vitreous loss, severe postoperative fibrinous uveitis is frequently expected in these patients. As a result of the intense and prolonged inflammation posterior synechiae, capsule contraction and cystoid macular edema may complicate the postoperative course of PXF patients. Topical NSAIDs after surgery have proved effective in reducing the amount of aqueous protein release and the percentage of patients showing increased macular thickness. ${ }^{52}$ Pressure spikes may occur

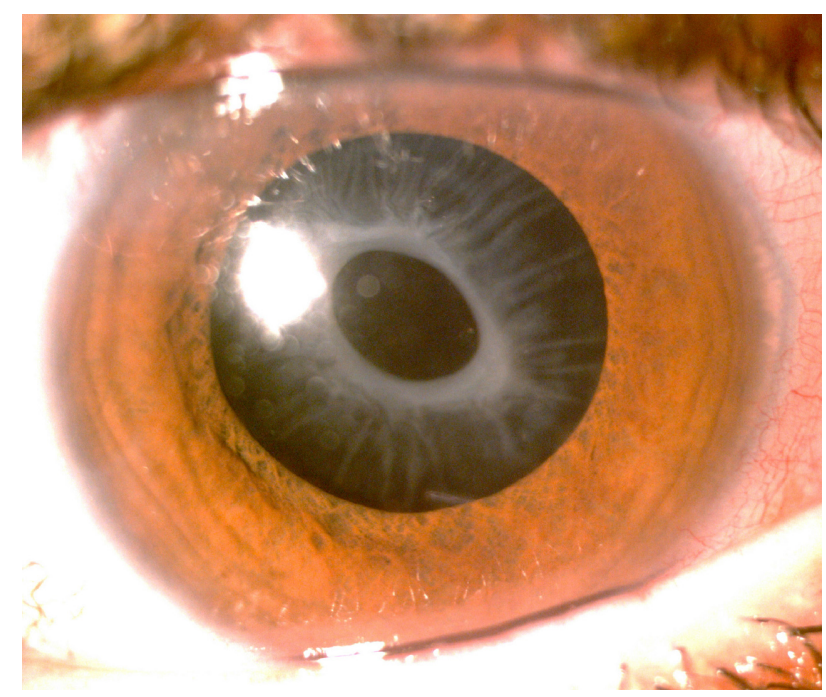

Figure 3 Slit lamp photograph showing anterior capsule opacification and phimosis after cataract surgery with in-the-bag IOL implantation.

Note: Note the reduction in size of the anterior capsulotomy with radial lines of capsular shrinkage, indicating traction on the peripheral lens zonules. Abbreviation: IOL, intraocular lens.

as result of aqueous protein release and are more frequently seen in patients with pre-existing glaucoma.

An increased risk of endothelial cells loss may be expected in PXF as a result of more complicated and prolonged surgery, often requiring complex manipulation of cataract, iris, and IOL. A three times greater percentage of endothelial cell loss is estimated in patients with PXF. ${ }^{25}$ However, despite the abnormal endothelium seen in these patients, the risk of irreversible corneal decompensation with surgery is low. ${ }^{19}$

Capsular phimosis with IOL decentration and late in-thebag IOL dislocation are among the most dreaded complications occurring after surgery (Figure 3). Capsular shrinkage determines centripetal traction on the weakened lens zonules, inducing tilt and decentration of the IOL with possible dislocation of the entire IOL-bag complex. Radial anterior relaxing Nd:YAG laser capsulotomy allows the release of the capsular traction over the peripheral zonules, thus potentially avoiding or delaying dislocation of the IOL in the vitreous cavity (Figure 4). Laser or surgical capsulotomy should be performed as soon as the capsular opening starts to shrink in order to avoid worsening of zonular damage. ${ }^{53}$

\section{Conclusion}

Cataract surgery in settings of PXF carries a significant risk of complications in the form of capsule rupture, vitreous loss, nucleus luxation, and IOL dislocation. PXF material involving most ocular tissues determines progressive weakening of 


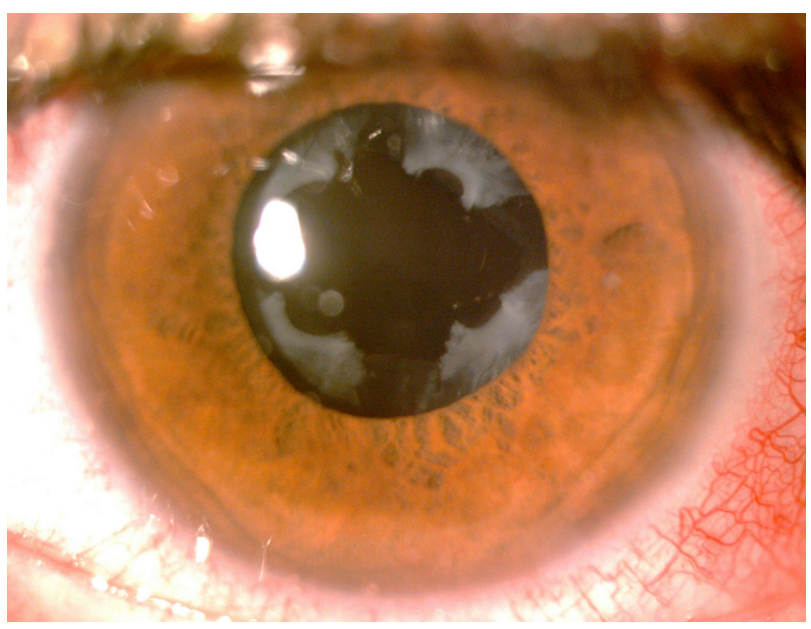

Figure 4 Slit lamp photograph after Nd:YAG laser anterior capsulotomy. Note: Lens capsule contraction was released with enlargement of the anterior capsulotomy.

the zonular ligament with consequent loss of lens stability. A planned approach to cataract surgery using the advanced techniques of phacoemulsification, specific OVDs, and capsule support devices significantly reduces the risk of complications during surgery. Postoperative surveillance is required to monitor and treat postoperative intraocular pressure, anterior chamber inflammation, IOL decentration, and cystoid macular edema.

Employing all these measures, cataract surgery can yield satisfactory visual outcomes in PXF patients.

\section{Disclosure}

The authors report no conflicts of interest in this work.

\section{References}

1. Bourne RR, Stevens GA, White RA, et al. Causes of vision loss worldwide, 1990-2010: a systematic analysis. Lancet. 2013;1(6):e339-e349.

2. Kanthan GL, Mitchell P, Burlutsky G, Rochtchina E, Wang JJ. Pseudoexfoliation syndrome and the long-term incidence of cataract surgery: the Blue Mountains eye study. Am J Ophthalmol. 2013;155(1): 83-88.e1

3. Ekström C, Botling Taube A. Pseudoexfoliation and cataract surgery: a population-based 30-year follow-up study. Acta Ophthalmol. 2015;93(8):774-777.

4. Schöltzer-Schrehardt U. Pseudoexfoliation syndrome: the puzzle continues. J Ophthalmic Vis Res. 2012;7(3):187-189.

5. Hiller R, Sperduto RD, Krueger DE. Pseudoexfoliation, intraocular pressure and senile lens changes in a population-based survey. Arch Ophthalmol. 1982;100(7):1080-1082.

6. Aasved H. The geographical distribution of fibrillopathia epitheliocapsularis, so-called senile exfoliation or pseudoexfoliation of the anterior lens capsule. Acta Ophthalmol (Copenh). 1969;47(3):792-810.

7. Thyghesen J. Prevalence and distribution of exfoliation syndrome. Glaucoma Topics Trends. 2006;4:5-6.

8. Aström S, Stenlund H, Lindén C. Incidence and prevalence of pseudoexfoliation and open-angle glaucoma in northern Sweden: II. Results after 21 years of follow-up. Acta Ophthalmol Scand. 2007;85(8):832-837.
9. Parekh P, Green WR, Stark WJ, Akpek EK. Electron microscopic investigation of the lens capsule and conjunctival tissues in individuals with clinically unilateral pseudoexfoliation syndrome. Ophthalmology. 2008;115(4):614-619.e2.

10. Conway RM, Schöltzer-Schrehardt U, Küchle M, Naumann GO. Pseudoexfoliation syndrome: pathological manifestations of relevance to intraocular surgery. Cin Exp Ophthalmol. 2004;32(2):199-210.

11. Schöltzer-Schrehardt UM, Koca MR, Naumann GO, Volkholz H. Pseudoexfoliation syndrome. Ocular manifestation of a systemic disorder? Arch Ophthalmol. 1992;110(12):1752-1756.

12. Schumacher S, Schöltzer-Schrehardt U, Martus P, Lang W, Nauman GO. Pseudoexfoliation syndrome and aneurysm of the abdominal aorta. Lancet. 2001;357(9253):359-360.

13. Mitchell P, Wang JJ, Smith W. Association of pseudoexfoliation syndrome with increased vascular risk. Am J Ophthalmol. 1997;124(5): 685-687.

14. Svensson R, Ekstrom C. Pseudoexfoliation and mortality: a population-based 30-year follow-up study. Acta Ophthalmol. 2015;93(2): $162-164$.

15. Thorleifsson G, Magnusson KP, Sulem P, et al. Common sequence variants in the LOXL1 gene confer susceptibility to exfoliation glaucoma. Science. 2007;317(5843):1397-1400.

16. Ritch R, Schlötzer-Schrehardt U. Exfoliation (pseudoexfoliation) syndrome: toward a new understanding. Proceedings of the first International Think Tank. Acta Ophthalmol Scand. 2001;79(2):213-217.

17. Vazquez-Ferreiro P, Carrera-Hueso FJ, Poquet Jornet JE, Fikri-Benbrahim N, Diaz-Rey M, Sanjuan-Cervero' R. Intraoperative complications of phacoemulsification in pseudoexfoliation: meta-analysis. J Cataract Refract Surg. 2016;42(11):1666-1675.

18. Walinder PE, Olivious EO, Nordell SI, Thoburn WE. Fibrinoid reaction after extracapsular cataract extraction and relationship to exfoliation syndrome. J Cataract Refr Surg. 1989;15(5):526-530.

19. Wirbelauer C, Anders N, Pham DT, Wollensak J. Corneal endothelial cell changes in pseudoexfoliation syndrome after cataract surgery. Arch Ophthalmol. 1998;116(2):145-149.

20. Moreno J, Duch S, Lajara J. Pseudoexfoliation syndrome: clinical factors related to capsular rupture in cataract surgery. Acta Ophthalmol (Copenh). 1993;71(2):181-184.

21. Bartholomew RS. Lens displacement associated with pseudocapsular exfoliation. Br J Ophthalmol. 1970;54(11):744-750.

22. Repo LP, Naukkarinen A, Paljarvi L, Terasvirta ME. Pseudoexfoliation syndrome with poorly dilating pupil: a light and electron microscopic study of the sphincter area. Graefes Arch Clin Exp Ophthalmol. 1996; 234(3):171-176.

23. Mardin CY, Schöltzer-Schrenhardt U, Naumann GO. "Masked" pseudoexfoliation syndrome in unoperated eyes with circular posterior synechiae: clinical-electron microscopic correlation. Arch Ophthalmol. 2001;119(10):1500-1503.

24. Rodriguez-Garcia A, Hernandez-Camarena JC, Lopez-Jaime GR, NavaGarcia JA. Effect of topical nonsteroidal anti-inflammatory drugs on pupillary size during uncomplicated cataract surgery. J Refract Surg. 2017;33(4):236-242.

25. Hayashi K, Manabe S, Yoshimira K, Kondo H. Corneal endothelial damage after cataract surgery in eyes with pseudoexfoliation syndrome. J Cataract Refr Surg. 2013;39(6):881-887.

26. Schöltzer-Schrehardt UM, Dörfler S, Naumann GO. Corneal endothelial involvement in pseudoexfoliation syndrome. Arch Ophthalmol. 1993; 111(5):666-674.

27. Naumann GO, Schöltzer-Schrehardt U. Keratopathy in pseudoexfoliation syndrome as a cause of corneal endothelial decompensation: a clinicopathologic study. Ophthalmology. 2000;107(6):1111-1124.

28. Asano N, Schöltzer-Schrehardt U, Naumann GO. A histopathologic study of iris changes in pseudoexfoliation syndrome. Ophthalmology. 1995;102(9):1279-1290.

29. Kückle M, Vinores SA, Mahlow J, Green WR. Blood-aqueous barrier in pseudoexfoliation syndrome: evaluation by immunohistochemical staining of endogenous albumin. Graefes Arch Clin Exp Ophthalmol. 1996;234(1):12-18. 
30. Quiroga L, Lansingh VC, Samudio M, Pena FY, Carter MJ. Characteristics of the corneal endothelium and pseudoexfoliation syndrome in patients with senile cataract. Clin Exp Ophthalmol. 2010; 38(5):449-455.

31. Yildrim N, Yasar E, Gursoy H, Colak E. Prevalence of pseudoexfoliation syndrome and its association with ocular and systemic diseases in Eskisehir, Turkey. Int J Ophthalmol. 2017;10(1):128-134.

32. Droslum L, Haaskjold E, Davanger M. Pseudoexfoliation syndrome and extracapsular cataract extraction. Acta Ophthalmol (Copenh). 1993;71(6):765-770.

33. Schöltzer-Schrehardt U, Naumann GO. Trabecular meshwork in pseudoexfoliation syndrome with and without open-angle glaucoma. A morphometric, ultrastructural study. Invest Ophthalmol Vis Sci. 1995;36(9):1750-1764.

34. Aström S, Lindén C. Incidence and prevalence of pseudoexfoliation and open angle glaucoma in northern Sweden: I. Baseline report. Acta Ophthalmol Scand. 2007;85(8):828-831.

35. Ekström C. Elevated intraocular pressure and pseudoexfoliation of the lens capsule as risk factors for chronic open-angle glaucoma. A population-based five-year follow-up study. Acta Ophthalmol (Copenh). 1993;71(2):189-195.

36. Vesti E, Kivelä T. Exfoliation syndrome and exfoliation glaucoma. Prog Retin Eye Res. 2000;19(3):345-368.

37. Kim S, Sung KR, Lee JR, Lee KS. Evaluation of lamina cribrosa in pseudoexfoliation syndrome using spectral-domain optical coherence tomography enhanced depth imaging. Ophthalmology. 2013;120(9):1798-1803.

38. Shingleton BJ, Laul A, Nagao K, et al. Effect of phacoemulsification on intraocular pressure in eyes with pseudoexfoliation: single surgeon series. J Cataract Refract Surg. 2008;34(11):1834-1841.

39. Kristianslund O, Østern AE, Råen M, Sandvik GF, Droslum L. Does cataract surgery reduce the long-term risk of glaucoma in eyes with pseudoexfoliation syndrome? Acta Ophthalmol. 2016;94(3):261-265.

40. Arshinoff SA. Dispersive-cohesive viscoelastic soft shell technique. J Cataract Refract Surg. 1999;25(2):167-173.

41. Hashemi H, Seyedian MA, Mohammadpour M. Small pupil and cataract surgery. Curr Opin Ophthalmol. 2015;26(1):3-9.

42. Wollensak G, Wollensak J. Double contour of the lens capsule edges after continuous curvilinear capsulorhexis. Graefes Arch Clin Exp Ophthalmol. 1997;235(4):204-207.
43. Greenburg DL, Coan EB. Capsule-splitting phenomenon during capsulorhexis in pseudoexfoliation. J Cataract Refract Surg. 2015; 41(6):1306-1309.

44. Jacob S, Agarwal A, Agarwal A, Agarwal S, Patel N, Lal V. Efficacy of a capsular tension ring for phacoemulsification in eyes with zonular dialysis. J Cataract Refract Surg. 2003;29(2):315-321.

45. Price FW Jr, Mackool RJ, Miller KM, Koch P, Oetting TA, Johnson AT. Interim results of the United States investigational device study of the ophthec capsular tension ring. Ophthalmology. 2005;112(3):460-465

46. Bayractar S, Altan T, Kucuksumer Y, Yilmaz OF. Capsular tension ring implantation after capsulorhexis in phacoemulsification of cataracts associated pseudoexfoliation syndrome; intraoperative complications and early postoperative findings. J Cataract Refract Surg. 2001;27(10):1620-1628.

47. Cionni RJ, Osher RH. Management of profound zonular dialysis or weakness with a new endocapsular ring designed for scleral fixation. J Cataract Refract Surg. 1998;24(10):1299-1306.

48. Shingleton BJ, Heltzer J, O’Donoghue MW. Outcomes of phacoemulsification in patients with and without pseudoexfoliation syndrome. J Cataract Refract Surg. 2003;29(6):1080-1086.

49. Mansour AM, Antonios RS, Ahmed II. Central cortical cleanup and zonular deficiency. Clin Ophthalmol. 2016;10:1919-1923.

50. Coca-Prados M. The blood-aqueous barrier in health and disease. J Glaucoma. 2014;23(Suppl 1):S36-S38.

51. Kuckle M, Nguyen NX, Hannappel E, Naumann GO. The bloodaqueous barrier in eyes with pseudoexfoliation syndrome. Ophthalmic Res. 1995;27(Suppl 1):136-142.

52. Coassin M, Iovieno A, Soldani A, et al. Bromfenac ophthalmic solution $0.09 \%$ as an adjunctive therapy to topical steroids after cataract surgery in pseudoexfoliation syndrome: a randomized clinical trial. J Cataract Refract Surg. 2016;42(8):1119-1125.

53. Liu E, Cole S, Werner L, Hengerer F, Mammalis N, Kohnen T. Pathologic evidence of pseudoexfoliation in cases of in-the-bag intraocular lens subluxation or dislocation. J Cataract Refract Surg. 2015;41(5): 929-935.
Clinical Ophthalmology

\section{Publish your work in this journal}

Clinical Ophthalmology is an international, peer-reviewed journal covering all subspecialties within ophthalmology. Key topics include: Optometry; Visual science; Pharmacology and drug therapy in eye diseases; Basic Sciences; Primary and Secondary eye care; Patient Safety and Quality of Care Improvements. This journal is indexed on Submit your manuscript here: http://www.dovepress.com/clinical-ophthalmology-journal

\section{Dovepress}

PubMed Central and CAS, and is the official journal of The Society of Clinical Ophthalmology (SCO). The manuscript management system is completely online and includes a very quick and fair peer-review system, which is all easy to use. Visit http://www.dovepress.com/ testimonials.php to read real quotes from published authors. 